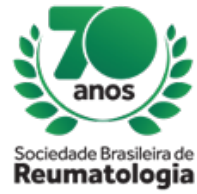

\title{
ANALYSIS OF ANTITHYROID ANTIBODIES AND THYROID HORMONES IN PATIENTS WITH SJOGREN'S SYNDROME.
}

Letícia Dall'Oglio Whitaker (Universidade do Sul de Santa Catarina, Palhoça, SC, Brasil), Anne Beatrice Crema Reginatto (Universidade do Sul de Santa Catarina, Palhoça, SC, Brasil), Maria Eduarda Dall'Oglio Whitaker (Universidade do Sul de Santa Catarina, Palhoça, SC, Brasil), Ivanio Alves Pereira (Universidade do Sul de Santa Catarina, Palhoça, SC, Brasil)

\section{BACKGROUND}

Other autoimmune diseases are common in patients with immune-mediated thyroid disorders. In patients with Sjogren's Syndrome the frequency of autoimmune thyroiditis and thyroid hormone dysfunction, as well as its association with the clinical and laboratory manifestations of the disease, need to be better studied. The objective was to evaluate the frequency and association of the presence of antithyroid antibodies and thyroid hormone dysfunction with the clinical and laboratory manifestations of SS.

\section{MATERIALS AND METHODS}

Retrospective observational study that included 174 patients with SS diseases according to the classification criteria ACR / EULAR 2016, attended from January 2000 to July 2017. Data were analyzed by SPSS 18.0 using chi-square $(x 2)$ for qualitative variables and Student $t$ test for quantitative variables, with significance at $p \leq 0.05$. The measure of association used was Prevalence Ratio (PR) with Confidence Interval $(95 \% \mathrm{Cl})$.

\section{RESULTS}

The presence of thyroid hormonal dysfunction was common, with hypothyroidism seen in $43.5 \%$ of the patients, whereas hyperthyroidism was seen only in $1.2 \%$. The thyroid autoantibody most commonly found was autoantibody of thyroglobulin (TgAb) present in $41.9 \%$ of patients, followed by antibody against thyroid peroxidase (TPO) in 35.8\%. It was found a significant association between hypothyroidism and anti-Ro / SSA $(p<0.01)$, anemia $(p=0.04)$ and similarly between low levels of free T4 and anti-Ro / SSA $(p=0.001)$, cutaneous manifestations $(p=0.04)$, parotitis $(p=0.01)$ and abnormal sialography $(p=$ $0.04)$.

\section{CONCLUSION}

Thyroid diseases, particularly hypothyroidism, are common in SS patients and are associated with the presence of anti-Ro / SSA and anemia. Thus, routine analysis of levels of thyroid stimulating hormone (TSH) and at least one thyroid antibody test associated with hypothyroidism, such as anti-TPO or TgAb, is important in the baseline evaluation of these patients. In addition to frequency, the clinical expression of various symptoms in thyroid pathologies, such as fatigue, anemia and polyarthralgia, may be confused with clinical complaints of SS. 